\title{
Mesenchymal stem cells and cartilage lesions
}

\author{
Jon Karlsson
}

Published online: 26 June 2013

(c) Springer-Verlag Berlin Heidelberg 2013

In this issue of the journal, Filardo et al. [1] give an overview of the available clinical evidence to support the use of mesenchymal stem cells in the treatment of cartilage injuries. They found that there is an increasing body of evidence-from both clinical and mostly preclinical studies-that mesenchymal stem cells might be the future when it comes to treating cartilage lesions.

There is obviously growing interest in various biological treatment alternatives today, and several treatment alternatives are commercially available. But, in spite of increased interest in biological treatment, we still have a long way to go. The above-mentioned study is a systemetic review, but most of the studies included were preclinical, and only 18 clinical studies on patients were found. Of these 18 studies, none were randomised and only 5 were comparative. Almost half of the studies were case reports. In other words, for the best possible clinical evidence we need randomised studies, either comparing the biological treatment alternatives with other well-known treatments, such as autologous cartilage implantation (ACI) or microfracture. Of course, we are facing problems in terms of good study design, in most cases due to the heterogeneous patient cohorts (age, gender, concomitnat injuries, etc.), making comparisons difficult or in some cases even impossible. There are still several unanswered questions, like the optimal cell source and the best way of delivery. Today, we base a lot of our knowledge on low-quailty studies and we-the researchers-must shape the future in a secure mode. Many of the studies are based on weak methodology, small cohorts, short follow-up time and only

J. Karlsson $(\bowtie)$

Department of Orthopaedics, Sahlgrenska University Hospital,

43180 Mölndal, Sweden

e-mail: jon.karlsson@telia.com limited reports of complications. We must walk on steady bones in the future in order to take care of our patients in the best possible manner.

What is important, however, is the growing interest in biological treatment approaches, and an increasing number of studies have been published during the last years. The growing interest is directed towards focal cartilage damage to frank osteoarthritis, with minimally invasive surgical approaches, either augmented or as growth factors or scaffolds. Several technical improvements have been developed during the last few years, and many of them are promising, at least in the short-term. Mesenchymal stem cells can be considered to be at least a promising approach, and the early clinical results are of interest in both the treatment of focal cartilage injuries and possibly osteoarthritis. With regards to osteoarthritis, we still have a long way to go. Also, we need to consider the risks; we know some of the risks but not all. For instance, the differentation of the mesenchymal stem cells into unwanted tissue is something that needs to be considered. In other words, instead of cartilage we might end up with bone. There are also some concerns about what can happen during the culture process of the mesenchymal stem cells.

What lies in the future? Today it is more or less obvious that the treatment of cartilage and other sportsrelated injuries with biological alternatives is still in its infancy and that more and better studies are needed; especially, we need to take the promising preclinical results from the laboratory to the patients. This can only be achieved with well-designed randomised controlled trials. We also need to gain increased knowledge about the natural history, but this is something that takes a long, long time, and we might not be prepared to wait. We need to understand many more details about the nature 
and funtion of the mesenchymal stem cells, like signalling factors. Cartilage is probably the most complex tissue in the body and to fully understand the repair process will take a lot of time and effort. But, using the current knowledge about mesenchymal stem cells, we might be walking on the right path.

\section{References}

1. Filardo G, Madry H, Jelic M, Roffi A, Cucchiarini M, Kon E (2013) Mesenchymal stem cells for the treatment of cartilage lesions: from preclinical findings to clinical application in orthopaedics. Knee Surg Sports Traumatol Arthrosc doi: $10.1007 / \mathrm{s} 00167-012-2329-3$ 